\title{
Prospects for a dominantly microwave-diagnosed magnetically confined fusion reactor
}

\author{
Francesco A. Volpe \\ Dept of Applied Physics and Applied Mathematics \\ Columbia University, New York, NY 10027, USA \\ fvolpe@columbia.edu
}

January 13, 2017

\begin{abstract}
Compared to present experiments, tokamak and stellarator reactors will be subject to higher heat loads, sputtering, erosion and subsequent coating, tritium retention, higher neutron fluxes, and a number of radiation effects. Additionally, neutral beam penetration in tokamak reactors will only be limited to the plasma edge. As a result, several optical, beam-based and magnetic diagnostics of today's plasmas might not be applicable to tomorrow's reactors, but the present discussion suggests that reactors could largely rely on microwave diagnostics, including techniques based on mode conversions and Collective Thomson Scattering.
\end{abstract}

\section{Introduction}

The international ITER tokamak [1] is expected to achieve a fusion gain (ratio of fusion power to input heating power) $Q=10$ and to demonstrate the scientific feasibility of magnetic confinement fusion (MCF).

The Fusion Nuclear Science Facility (FNSF) 2, 3, 4] is a tokamak or spherical tokamak advocated in the United States, with emphasis on nuclear issues and on the integration of the first wall, blanket, shield, vacuum vessel and divertor in the nuclear fusion environment.

DEMO [5] is the common name for the fusion device -most likely a tokamak or a stellarator- expected to demonstrate the technological feasibility of MCF.

ITER, FNSF, DEMO and a magnetic confinement fusion power plant will operate longer and longer plasma discharges and will generate larger and larger amounts of fusion power, in absolute terms as well as normalized to input power. Correspondingly, as they are all expected to be based on D-T reactions, they will generate larger and larger fluences of $14.1 \mathrm{MeV}$ neutrons, resulting in increased material damage, as summarized in Table 1 First walls, blankets and coolants will also be hotter, as hot as about $600^{\circ} \mathrm{C}$ in a reactor. This poses special requirements on materials [6] and diagnostics [7, 8, 9, 10, 11, 12, 13, 14, 15, 16].

MCF reactors will not be physics experiments and, apart from initial scenario optimizations and periodic calibrations, they will not require several engineering or physics parameters to be scanned. Instead, they will be steadily operated in a smaller parameter space. Yet, precisely for this reason, plasma parameters will need to be continuously diagnosed with sufficient temporal and spatial resolution to prevent undesired instabilities as well to maintain optimal conditions (density, temperature, rotation, field errors, plasma shape, position and others) for confinement, fusion reactivity etc. For these reasons, it is important to develop reactor-grade diagnostics and ensure all relevant measurements. In decreasing order of importance, these are: measurements for machine protection and basic control, measurements for advanced control, and those for performance evaluation [7, 9, 17.

At the same time, all measurements will have to be compatible with the harsh fusion reactor environment. That is, they will have to face the issues listed in Sec 2 of the present paper, distilled from the vast literature on the subject $[7,8,9,10,11,12,13,14,15,16$. . Optical and inductive diagnostics will be particularly susceptible to such issues, and neutral beams will only be available in the outer part of the plasma. Possible countermeasures include shielding the diagnostics and investigating new materials (Sec 3) or relying as extensively as possible on microwave diagnostics that, despite their own issues [18]-[39, are more resilient to the reactor environment (Sec44).

With this preamble, the present paper discusses for the first time the question of whether it will be possible to measure all or nearly all quantities of interest in a reactor by means of microwaves. The answer to this question is encouraging: as discussed in Sec 5.1 and summarized in Table 2 of the present paper, more than two thirds of all the relevant observables listed in Refs. 7, 9, 17, are directly measurable by either well-proven or less established but promising microwave techniques -based for example on Collective 
Table 1: Maximum damage, in displacements per atom (dpa), and typical plasma discharge duration expected in future MCF devices [3, 4].

\begin{tabular}{|lllll|}
\hline & ITER & FNSF & DEMO & Power Plant \\
\hline Max damage & $3 \mathrm{dpa}$ & $37-74$ dpa & $100-150$ dpa & $>150$ dpa \\
Plasma discharge duration & $500-3000 \mathrm{~s}$ & $1-15$ days & $15-365$ days & $>365$ days \\
\hline
\end{tabular}

Thomson Scattering, Electron Bernstein Waves or mode conversions. Emphasis is laid on the physical principles enabling the measurements.

Another goal of the present article is to identify research needs and future directions: as summarized in Table 3, there are still several quantities that, at present, can only be measured indirectly by microwaves (Sec 5.2 ), or not at all (Sec 5.3 ), but it would be highly desirable to directly measure them by means of microwaves. It goes beyond the scope of the present paper to review established microwave diagnostics and past results. For that purpose, the reader is redirected to a book [4] and review papers [41, 42, 43].

\section{Diagnostic challenges in a MCF reactor}

Diagnostics in a reactor will face [12, 13, 16, 14]:

- High heat loads.

- High fluences of energetic particles, leading to sputtering and erosion. Among others, this leads to the coating of optics by impurities and polymers [4, 45, 46, 47, 48, 49.

- High fluences of $14.1 \mathrm{MeV}$ neutrons, carrying $80 \%$ of the power produced in D-T fusion reactions. At hundreds or thousands of MW, and divided by the wall surface, this implies exposure of the walls to about $1 \mathrm{MW} / \mathrm{m}^{2}$. Significant amounts of $2.45 \mathrm{MeV}$ D-D neutrons will also be produced. This results in "nuclear heating" [50] of the walls and underlying materials, as well as in transmutations [51. The latter affect semiconductors by transmuting the bulk material or the dopant into another element with different donor or acceptor properties [52, 53].

- Radiation-induced electromotive force due to Compton and photo-electrons [54, 55, 56. This electromotive force can lead to spurious voltages along as well as across mineral-insulated coaxial cables. Note that other insulations (for example, plastic) would be radiation-damaged too quickly. Inductive diagnostics will suffer from such issues.

- Radiation-induced degradation or alteration of material properties. This includes:

- The introduction of finite conductivity in insulators [57, [58, 59, 8], affecting inductive diagnostics of magnetic field, bolometers and pressure gauges.

- The darkening of refractive optics [60. This is a volume effect due to radiation, distinct from superficial sputtering and erosion effects.

- Radiation-induced luminescence (radioluminescence) in windows and optical fibers [61, 62, 59, 8.

- Damage of solid-state components and detectors [63, 64 as used in infrared, visible, ultraviolet, $\mathrm{X}$-ray and neutron cameras, which can be contained by proper doping and radiation modification [52].

- Tritium retention issues [65], for example in spectrometers [66].

- Vibrations and large electromagnetic forces, introducing statistical and systematic errors in diagnostic alignment.

- Thermoelectric effects, whether radiation-induced [67, 68] or not [69, resulting in a thermally induced electromotive force.

- Reduced neutral-beam penetration [70, 71, 72] due to the large minor radius, especially in a tokamak reactor. This might affect beam-based diagnostics, restrict their applicability to the plasma edge and prevent the core from being diagnosed. In addition, the signal-to-noise ratio is expected to degrade as a result of the high density $n$ and large minor radius $a$. This is because the signal grows like $n$, but Bremsstrahlung grows like $n^{2} a$ [72]. Diagnostics affected include: Beam Emission Spectroscopy (BES), measuring electron density fluctuations [73; Charge Exchange Recombination (CER), measuring ion temperatures and flow velocities [74; Motional Stark Effect (MSE), measuring the magnetic field rotational transform [75]. All three diagnostics are highly relevant to tokamak confinement and stability, but the last two are less needed in stellarators.

- Tokamak reactors will have additional needs and challenges compared with stellarator reactors, due to disruptions and runaway electron beams. 
If follows from this list that several diagnostics commonly used in present tokamaks might have issues or be unavailable in a reactor. Beam penetration issues will affect BES, CER, MSE and Lithium beam spectroscopy. Sputtering and coating of windows and mirrors, and volumetric radiation damage to optical materials will affect optical diagnostics such as Thomson Scattering, Survey Poor Resolution Extended Domain (SPRED) spectroscopy, visible Bremsstrahlung and other spectrometers, filterscopes, phase contrast imaging, infrared, visible and ultraviolet cameras, including fast cameras. Radiation damage, including neutron damage, will affect inductive magnetic diagnostics, bolometers and pressure gauges. This and thermal damage would affect diagnostics in close proximity to the plasma and first wall, such as tile-embedded thermocouples, or even in direct contact with the plasma edge, such as Langmuir probes.

\section{Diagnostic protection and new materials}

One could think of several strategies to counteract the issues above.

One possible strategy is obviously to protect existing diagnostics from neutrons and radiation in general (by means of shielding), and from exposure to plasma, energetic particles and stray electromagnetic waves (e.g. by means of shutters, to limit their utilization to when strictly necessary). Cooling can protect from high heat-loads and heat-transients.

Even with these precautions, however, survival in DEMO would be limited to about three months for magnetics, one week for bolometry and few hours for pressure gauges and VUV windows, and this is only accounting for radiation effects in DEMO [17]; survival in a plant, and with all effects included, would be even shorter. Also note that laser- and microwave-based techniques for in-situ cleaning of windows, lenses and mirrors 45, 47, 48, 49, while useful and indispensable in ITER, do not prevent volumetric damage by neutrons, especially in DEMO or a power plant.

The next level of protection is to identify new materials for existing techniques. New materials are being sought, which are more resilient to radiation effects and transmutations. Platinum, in this sense, is a good replacement for gold [76, 77]. Single-crystal [78] and liquid mirrors [79] are being considered, because radiation-harder than regular mirrors.

Inevitably, though, lenses and mirrors will be damaged, which implies that their use should be minimized, and direct lines of sight should be adopted as much as possible.

\section{Advantages and disadvantages of microwave diagnostics}

Another approach is complementary and possibly alternative to the diagnostic protection and material innovations just mentioned in Sec 3 . The idea is to rely on long wavelengths such as far infrared, THz, millimeter waves and microwaves: in proportion with the longer wavelength, diagnostics are less sensitive to the polishing and finish of windows, mirrors and lenses, and less sensitive to sputtering and erosion damage. The "grooves" and rough features resulting from sputtering, erosion and other damage are typically much smaller, both in width and depth, than the wavelength and skin-depth $\delta=\sqrt{2 \rho / \omega \mu}$. Here $\rho$ is the bulk resistivity and $\mu=\mu_{0} \mu_{r}$ the magnetic permeability. For frequencies $\omega / 2 \pi=1-100 \mathrm{GHz}$, relative permeability of the medium $\mu_{r} \simeq 1$ and typical metal resistivities, the skin depth amounts to $0.2-7$ $\mu \mathrm{m}$.

Another effect is coating: material sputtered elsewhere can deposit on microwave optics. However, when such material is dielectric, its effect on microwave attenuation, for typical coating thicknesses of few $\mu \mathrm{m}$ or less, refractive index $N=1-2$ and loss tangent of $10^{-5}-10^{-2}$ [80, is negligible. Reflectivity can deteriorate due to dielectric material deposition, but mostly in the utraviolet and visible range; the reflectivity of dielectric materials in the microwave range is comparatively much higher [81. A metalic film, on the other hand, can have a significant shielding effect and make a window or a lens opaque, in so far as it is thicker than the skin depth and "holes" in the coating are not bigger than the wavelength. The effect on microwave mirrors, instead, is probably negligible: the reflectivity of the metallic film deposited might be lower than that of the metal underneath, but only by few percents [81, which are easily accounted for in a recalibration.

The adoption of even longer wavelengths, thus lower frequencies $(\lesssim 500 \mathrm{MHz})$ is probably to be avoided, because antennas and inductive coils face other issues related to cable damage (see Sec 2 ).

That being said, reactor-grade microwave diagnostics will not be immune from practical issues either [18. For instance, antennas will need to be carefully designed and positioned, and antennas' arrays carefully configured [18, with the aid of ray-tracing, beam-tracing [19] and full-wave codes [20, 21] as to collect acceptable signals despite refraction and vertical movements of the plasma. Concerns on limited access led to proposals of adopting a single spherical mirror 22] or Rowland circle optics [23] for microwave imaging. Windows and other in-vessel and in-port components will have to withstand microwave stray radiation [24, 25], electromagnetic loads, neutron activation, and meet maintenance, remote handling and safety requirements $[26,27$. They will also have to be properly designed to enable diagnostic calibration [28, 29]. Transmission lines will be longer than in present devices and will require high mode purity and low 
losses and reflections 30, 31. Finally the magnetic field will be higher, thus Electron Cyclotron Emission (ECE), Collective Thomson Scattering and other diagnostics will require sources [32] and receivers 33] to operate at higher frequencies.

Yet, these issues are considered solved or solvable in the conceptual and detailed designs of the ITER ECE radiometer [34, 35, 36, reflectometers [37] and other microwave diagnostics [9]. All things considered, even in a reactor, these issues are less challenging than those to be faced by non-microwave diagnostics, listed above. Microwave signals might be difficult to interpret due for instance to the relativistic downshift of the emission frequency [38, 39], but at least they will be available, unlike others, and they are expected to offer satisfactory signal-to-noise ratio, spatial and temporal resolution [34, 35, 36, 37, 9, 39.

\section{Important observables and their microwave and non-microwave diagnostics}

Refs. [7, 9, 17] compiled a table of measurements needed in ITER and, with good approximation, in a reactor. Diagnostics were grouped by their purpose, in decreasing order of urgency: 1a) machine protection and basic control, 1b) advanced control and 2) performance evaluation and physics.

That table is reproduced in Tables 2 and 3 here, with the additional classification of measurability by microwaves, which can be direct (Sec 5.1), indirect (Sec 5.2$)$ or pose a possible research need (Sec 5.3).

\subsection{Direct measurements by microwaves}

Microwave emission, interference, reflection, refraction and scattering provide information on the local or line-averaged dielectric tensor $\epsilon$, or selected components. These contain information on the electron density $n_{e}$, temperature $T_{e}$, mean flow, and magnetic field [40, 41, 42, 43].

Some microwave measurements are well known and well established [40, 41, 42, 43. These include $T_{e}$ profile measurements by Electron Cyclotron Emission (ECE) and $n_{e}$ measurements by interferometry. The latter are line-intergrated, but profiles can be inverted from multi-chord measurements. These diagnostics can also measure $n_{e}$ and $T_{e}$ fluctuations, thanks to their high temporal resolution or to related concepts such as Correlation ECE (CECE) [82, 83, 84.

Some microwave diagnostics can measure magnetic fields. Examples include polarimetry by Faraday rotation [85] or Cotton-Mouton effect [86. Both techniques are line-integrated and both are sensitive to density and to a magnetic field component (transverse and parallel to the line of sight, respectively) [87. They can thus measure that field component, provided that the density profile is known otherwise.

Here, however, it is more useful to discuss less common microwave techniques and discuss how they can fill the gaps to be left in a reactor by inductive, optical, atomic-beam diagnostics, and others.

Starting with measurements needed for machine protection and basic control (group 1A in Refs. [7, 9, 17] and Table 2, some of the observables "at risk" are plasma shape and position, separatrix-wall gaps and the gap $\delta_{\text {sep }}$ between the separatrices for the upper and lower null. Traditionally these quantities are reconstructed by EFIT [88] or other equilibrium reconstruction code on the basis of magnetic-probe and saddle-loop measurements. However, more recently the radial position of the plasma was measured reflectometrically, and adjusted in feedback with that measurement [89, 90]. The cutoff density and thus the microwave frequencies are lower than those used for density profile measurements. Several reflectometers operating at slightly different frequencies, as to not cross-talk with each other, could locate the separatrices at various poloidal locations, for $2 \mathrm{D}$ equilibrium reconstructions. With the addition of toroidally displaced reflectometers, 3D equilibria could be reconstructed as well.

The ionization front position in the divertor can also be measured reflectometrically.

Runaway electrons form tails in the electron distribution function, which can be measured by oblique [91, 92, 93, 94, 95, or vertical ECE [96, 97, 98.

Additional plasma parameters can be directly measured by Collective Thomson Scattering, modeconversion-based and other scattering diagnostic, as discussed in Secs 5.1.1.5.1.3.

\subsubsection{Collective Thomson Scattering}

Collective Thomson Scattering (CTS) 99, 100] is the scattering of electromagnetic waves off the electrons in the Debye spheres associated with ions. Thus, the scattered waves contain information on the ions, provided that the Salpeter parameter $\alpha=1 /\left|\mathbf{k}_{s}\right| \lambda_{D}$ is larger than 1 . Here $\mathbf{k}_{s}$ is the scattering wavevector and $\lambda_{D}$ the Debye length. Unless a back-scattering geometry is adopted, which has the drawback of poor spatial resolution, the criterion $\alpha>1$ translates in the requirement for low frequencies. Typical frequencies are $70-250 \mathrm{GHz}$, provided that the plasma is underdense to them. A CTS source must satisfy additional requirements of narrow spectrum, high power (as a consequence of the small scattering cross-section) and long pulses or continuous operation [101, 102]. The typical source of choice is the gyrotron, although the Cyclotron Autoresonance Maser recently regained attention 32. 
Table 2: Measurements needed in ITER and, with good approximation, in a reactor, adapted from Refs. [7, 9, 17. The observables are categorized by urgency and by the capability of microwave diagnostics to directly or indirectly measure them. See also Table 3 .

\begin{tabular}{|c|c|c|c|}
\hline $\begin{array}{l}\text { Microwave } \\
\text { meas. capa- } \\
\text { bility }\end{array}$ & $\begin{array}{l}\text { Group 1A } \\
\text { Meas. for machine pro- } \\
\text { tection and basic con- } \\
\text { trol }\end{array}$ & $\begin{array}{l}\text { Group 1B } \\
\text { Measurements for ad- } \\
\text { vanced control }\end{array}$ & $\begin{array}{l}\text { Group } 2 \\
\text { Performance evaluation } \\
\text { and physics }\end{array}$ \\
\hline $\begin{array}{l}\text { Direct } \\
\text { (Secs.5.1 } \\
\text { and } 5.1 .3\end{array}$ & $\begin{array}{l}\text { - Plasma shape and } \\
\text { position, } \\
\text { separatrix-wall gaps, } \\
\text { gap between } \\
\text { separatrices } \\
\text { - Line-averaged } \\
\text { electron density } \\
\text { - Runaway electrons }\end{array}$ & $\begin{array}{l}\text { - Plasma rotation } \\
\text { (toroidal and poloidal) } \\
\text { - Electron temperature } \\
\text { profile (core) } \\
\text { - Electron density } \\
\text { profile (core and edge) } \\
\text { - Ionization front } \\
\text { position in divertor } \\
-n_{e} \text { of divertor plasma }\end{array}$ & $\begin{array}{l}\text { - } T_{e} \text { profile (edge) } \\
-n_{e}, T_{e} \text { profiles } \\
\text { (X-point) } \\
-T_{e} \text { fluctuations } \\
-n_{e} \text { fluctuations } \\
\text { - Edge turbulence }\end{array}$ \\
\hline $\begin{array}{l}\text { Direct, by } \\
\text { CTS } \\
\text { (Sec 5.1.1 }\end{array}$ & $\begin{array}{l}\text { - Impurity and } \mathrm{D}, \mathrm{T} \\
\text { influx (divertor \& main } \\
\text { plasma) } \\
\text { - } Z_{\text {eff }} \text { (line-averaged) } \\
\text { - } n_{T} / n_{D} \text { in plasma core }\end{array}$ & $\begin{array}{l}\text { - } \alpha \text {-source profile } \\
\text { - Helium density profile } \\
\text { (core) } \\
\text { - Ion temperature } \\
\text { profile (core) } \\
\text { - } Z_{\text {eff profile }} \\
\text { - Helium density } \\
\text { (divertor) } \\
\text { - Impurity density } \\
\text { profiles } \\
-\alpha \text {-particle loss }\end{array}$ & $\begin{array}{l}\text { - } T_{i} \text { in divertor } \\
\text { - Confined } \alpha \text { particles } \\
\text { - } n_{T} / n_{D} / n_{H} \text { (edge) } \\
-n_{T} / n_{D} / n_{H} \text { (divertor) }\end{array}$ \\
\hline $\begin{array}{l}\text { Direct, } \\
\text { by mode } \\
\text { conversions } \\
\text { (Sec 5.1.2 }\end{array}$ & - Plasma current, $q_{95}$ & $\begin{array}{l}\text { - Current density } \\
\text { profile ( } q \text {-profile) } \\
\text { - Low } m / n \text { MHD } \\
\text { activity } \\
-T_{e} \text { of divertor plasma }\end{array}$ & - TAE modes, fishbones \\
\hline $\begin{array}{l}\text { Indirect } \\
\text { (Sec } 5.2\end{array}$ & $\begin{array}{l}\text { - Fusion power } \\
\text { - } \beta_{N}=\beta_{t o r}\left(a B / I_{p}\right) \\
\text { - Disruption precursors } \\
\text { (locked modes, } m=2) \\
\text { - H/L mode indicator } \\
\text { - ELMs }\end{array}$ & - Sawteeth & $\begin{array}{l}\text { - MHD activity in } \\
\text { plasma core }\end{array}$ \\
\hline
\end{tabular}

The CTS spectrum mimics the ion distribution function [103, or a convolution of ion distribution functions if multiple species are present, due to impurities, to two fuel ions (D and T) and due to fusion $\alpha$ 's.

If the ions of the main species are Maxwellian, CTS can measure their temperature $T_{i}$ [104. It can also measure related quantities, such as flows in the direction of the scattering vector, simply resulting in a shift of the said distribution [105, 106].

The convolution is easier to separate if the species of interest have dramatically different ion velocity distributions, due for example to very different masses and/or energies. This implies that CTS can measure the slowdown of $\alpha$ 's due to collisions, to radiations and to other effects [106, 107, the concentration of high $Z$ impurities, and $Z_{\text {eff }}$ [108, 109]. In principle, for sufficiently high precision (requiring an intense source and a low-noise radiometer), CTS can also measure the ion ratio $n_{T} / n_{D}$ or $n_{T} / n_{D} / n_{H}$, and core density of He ashes [107, 109].

All measurements are easily resolved in space, by crossing the incident microwave beam with several receiver beams. That is, profiles can be easily acquired of all the said quantities, and studied as a function of time, enabling transport studies of $\alpha$ particles and distinction between confined and lost $\alpha$ 's.

Perturbative, time-resolved experiments show promise for transport studies, e.g. of D, T and impurity influx. 


\subsubsection{Mode conversion based techniques}

The O-X mode conversion can be used to locally diagnose the field $\mathbf{B}$ and related quantities such as the edge safety factor $q_{95}=a B_{T} / R B_{p}$. Here $a$ and $R$ are the plasma minor and major radius, and $B_{T}$ and $B_{p}$ are the toroidal and poloidal field. From $B_{p}$ one can determine the plasma current $I_{p}=\mu_{0} B_{p} / 2 \pi a$, typically measured by Rogowski coils. In fact, good part of the $q$ profile (or, equivalently, current profile) can be measured by mode conversion based techniques, offering a complement and possible replacement for the optical, beam-based MSE [75] and other non-microwave techniques [110, 111, 112, 113, 114].

The basic idea is that a special $\mathbf{B}$-dependent view makes the $\mathrm{O}$ and $\mathrm{X}$-modes degenerate and not evanescent at the $n$-dependent O-mode cutoff layer. The angular map of conversion efficiency around that optimal direction also contains information on the local B: the inclination of the conversion contours at various frequencies $f$ gives the inclination of field-lines at various radial locations.

This was confirmed by two-dimensional scans 115, 116, including rapid scans performed during a single discharge by means of a spinning mirror [116], and eventually measurements simultaneously carried out with multiple sensors [17]. These initial demonstrations required the plasma to be overdense and emit Electron Bernstein (B) Waves (EBWs) that converted to the X-mode and eventually to the O-mode.

The more recent proposal and simulation of oblique reflectometry imaging [118, shows that the O-X conversion of an externally injected wave has advantages over the B-X-O conversion of internally emitted EBWs. The idea is now that, instead of a peak in transmissivity, the diagnostic characterizes a minimum in reflectivity, obtained for the same special, B-dependent direction. This is more flexible and can be operated at arbitrary densities and fields, as it does not require the plasma to be an overdense EBW emitter. The signal-to-noise ratio is also superior, by adopting a sufficiently intense external reflectometric source. The spatial and temporal resolution are reflectometer-like (of the order of $\mathrm{mm}$ and sub-ms), more than sufficient for a reactor.

As a consequence of measuring the $\mathbf{B}$ components along a fixed chord with good sensitivity, spaceand time-resolution, the diagnostic is expected to be sensitive to oscillations of $\mathbf{B}$ associated with rotating modes in the plasma, such as tearing modes of low poloidal/toroidal mode numbers $m / n$, Toroidal Alfvén Eigenmodes (TAEs) and fishbones.

Future modeling might clarify whether this technique could also detect and characterize non-rotating magnetic islands and other modes in the plasma, for example from the deformation or displacement of the conversion efficiency contours, or field stochasticity, from fluctuations and distortions in the contours.

The O-X conversion is sufficient for the technique just described. In principle, B-X-O converted EBW emission could also be of use in reactors, to measure $T_{e}$ in overdense plasmas not accessible to conventional ECE. It should be noted that at the very high field of a reactor, $B_{T} \geq 5 \mathrm{~T}$, the fundamental EC harmonic becomes inacessible at a very high density, $n_{e} \geq 2.4 \cdot 10^{20} \mathrm{~m}^{-3}$, which is unlikely in a tokamak. However, these high densities could be achieved in a stellarator high density H-mode [119.

Another mode conversion based technique of potential use in a reactor is EBW emission from the underdense edge and divertor region [120. Measurements of $T_{e}$ in this optically thin region might be difficult by conventional ECE. However, the plasma is optically thick to EBWs. Note that EBW emission and propagation do not require the plasma to be overdense (only the B-X-O conversion does). The only requirement to couple EBWs with external electromagnetic waves is for the Upper Hybrid layer (but not necessarily the O-mode cutoff) to lie in the plasma. This condition is met by wave frequencies $\omega \approx \omega_{c e}$, where $\omega_{c e}$ is the electron cyclotron frequency in the divertor region. EBW emission could be extracted by B-(SX)-FX conversion [121, where SX and FX refer to the slow and fast X-mode.

\subsubsection{Other scattering techniques}

Microwave scattering also assures turbulent fluctuations of electron density $n_{e}$, through Doppler broadening of sharp lines (much narrower than $1 \mathrm{MHz}$ ) in the tens of $\mathrm{GHz}$ range of frequencies. This led to measurements of electron temperature gradient (ETG) turbulence [122. The shift of the scattered spectrum relative to the incident line informs about the electron diamagnetic drift or, more generally, flows, including plasma rotation.

Cross-Polarization Scattering 123 , as the name suggests, examines scattering in which an incident O-mode (or X-mode) changes its polarization to X-mode (or O-mode). These changes of polarization are due to magnetic field fluctuations $\delta B$ in the plane perpendicular to $\mathbf{k}$. The actual modulus of $\mathbf{B}$, however, or its components, are unknown.

Another technique 124 aims at measuring the magnetic pitch angle based on the fact that turbulent structures are elongated in the pitch angle direction. In turn, such structures can be visualized by imaging scattered or reflected microwaves.

\subsection{Indirect measurements by microwaves}

The evolution of the pedestal, Edge Localized Modes (ELMs), the distance from the peeling-ballooning stability boundary, the quiescent H-mode, I-mode and other pedestal physics can simply be studied by 
Table 3: Measurements needed in ITER and, with good approximation, in a reactor, but currently not possible by microwave techniques (Sec.5.3). Adapted from Refs. [7, 9, 17]. See also Table 2.

\begin{tabular}{|c|c|c|c|}
\hline & $\begin{array}{l}\text { Group 1A } \\
\text { Meas. for machine pro- } \\
\text { tection and basic con- } \\
\text { trol }\end{array}$ & $\begin{array}{l}\text { Group 1B } \\
\text { Measurements for ad- } \\
\text { vanced control }\end{array}$ & $\begin{array}{l}\text { Group } 2 \\
\text { Performance evaluation } \\
\text { and physics }\end{array}$ \\
\hline Divertor & $\begin{array}{l}\text { - Surface temperature } \\
\text { (divertor \& upper } \\
\text { plates) } \\
\text { - Divertor detachment } \\
\text { indicator } \\
\text { - } j_{\text {sat }}, n_{e}, T_{e} \text { at divertor } \\
\text { plate }\end{array}$ & $\begin{array}{l}\text { - Heat deposition } \\
\text { profile (divertor) } \\
\text { - Net erosion (divertor } \\
\text { plate) }\end{array}$ & \\
\hline Gas & $\begin{array}{l}\text { - Gas pressure (divertor } \\
\text { \& duct) } \\
\text { - Gas composition } \\
\text { (divertor \& duct) }\end{array}$ & $\begin{array}{l}\text { - Neutral density } \\
\text { between plasma and } \\
\text { first wall }\end{array}$ & \\
\hline Radiation & $\begin{array}{l}\text { - Radiated power (main } \\
\text { plasma, X-point, diver- } \\
\text { tor) }\end{array}$ & $\begin{array}{l}\text { - Radiated power pro- } \\
\text { file (core, X-point, di- } \\
\text { vertor) }\end{array}$ & \\
\hline Neutrons & & $\begin{array}{l}\text { - Neutron profile } \\
\text { - Neutron fluence }\end{array}$ & \\
\hline $\begin{array}{l}\text { Electro- } \\
\text { magnetic }\end{array}$ & $\begin{array}{l}\text { - Loop voltage } \\
\text { - Halo currents }\end{array}$ & & $\begin{array}{l}\text { - Radial electric field } \\
\text { and field fluctuations }\end{array}$ \\
\hline Other & $\begin{array}{l}\text { - Surface temperature } \\
\text { (first wall) } \\
\text { - Dust }\end{array}$ & & \\
\hline
\end{tabular}

ECE, possibly combined with multi-chord interferometry.

The normalized beta, $\beta_{N}=\beta_{\text {tor }} a B / I_{p}$, can be indirectly measured from the prescribed magnetic field $B$ and from the microwave measured minor radius $a$, plasma current $I_{p}$ and $\beta_{t o r}$, which, in turn, is defined as the ratio between the measured kinetic pressure $k_{B}\left(n_{e} T_{e}+n_{i} T_{i}\right)$, where $k_{B}$ is the Boltzmann constant, and prescribed magnetic pressure $B_{T}^{2} / 2 \mu_{0}$. Similar considerations apply to the stored plasma energy and to the poloidal beta, $\beta_{p}$.

The fusion power in a D-T reactor equals five times the energy associated with the $\alpha^{\prime} s$, measurable by CTS, divided by the energy confinement time, which can be perturbatively inferred from microwavemeasured profiles of $n_{e}, n_{i}, T_{e}, T_{i}$.

Finally, direct microwave measurements of magnetic field components, e.g. associated with rotating modes, were discussed in Sec5.1.2 but it should be added that MHD activity can also be measured indirectly, from the associated temperature fluctuations, which can be measured by ECE. Examples include disruption precursors such as the rotating $m=2$ mode, sawteeth, and core MHD, not accessible by oblique reflectometry imaging. The diagnosis of non-rotating ("locked") MHD requires arrays of ECE radiometers, or ECE imaging.

\subsection{Observables difficult to measure by microwaves, future research needs}

Some measurement objectives are not microwaves, "by definition". These include observables at different wavelengths, such as visible line-emission or X-ray Bremsstrahlung, or simply broadband-integrated measurements of radiation. They also include other particles besides photons -mostly fusion neutrons.

Measuring such observables by means of microwaves would require up- or down-conversion from different frequencies to microwaves. Unfortunately this is not a very common need; conversion to visible light (for example from X-rays, by means of a CsI (Tl) scintillator, or from infrared, by upconversion nanoparticles) is in much higher demand.

Neutron measurements would require scintillators that are sensitive to neutrons and emit in the microwave or far infrared range and, at the same time, are resilient to radiation damage, probably amorphous (plastic or liquid), and resilient to transmutations.

Measurements of gas pressure and composition represent another diagnostic gap in a reactor (Table 3). It will be important to develop alternatives to conventional pressure gauges and multi gas analyzers (MGAs), bearing in mind that infrared and microwave spectroscopy are sensitive to vibrational and 
rotational transitions, respectively, in molecules. Atomic spectroscopy requires visible and ultraviolet light.

The need for innovative gas diagnostics is particularly strong in the divertor region. Other innovations are needed for the thermal and surface characterization of the divertor plates, as well as to measure $n_{e}$, $T_{e}$ and the ion-saturation current-density $j_{\text {sat }}$ in the nearby plasma (Table 3 ).

The challenges to be faced by magnetic diagnostics were described in Sec2. Innovations might be needed in diagnosing the loop voltage, typically measured by flux loops, and halo currents during disruptions, typically measured by Rogowski and segmented Rogowski coils [125].

Dust presence and dust dynamics is also of concern, e.g. for fear of disruptions [126]. These and other quantities, currently not measurable by microwave techniques, are listed in Table 3

\section{Summary and conclusions}

Magnetic confinement fusion reactors will not be physics experiments and, apart from initial scenario optimizations and periodic calibrations, for the most part they will be steadily operated at constant, optimal parameters for confinement, stability and fusion reactivity. Yet, precisely for this reason, plasma parameters will need to be continuously diagnosed with sufficient temporal and spatial resolution to maintain such optimal conditions.

Unfortunately, however, today's magnetic, optical and beam-based diagnostics will face various challenges in the harsh reactor environment. Microwave and direct-line-of-sight diagnostics, on the other hand, are more robust. For these reasons, in parallel with ongoing research on new materials, radiation hardening, neutron shielding and in-situ cleaning of existing diagnostics, it is estimated that new and existing microwave techniques could diagnose more than half of the relevant observables (Tables 2 and 3. Among others, a more extensive use of reflectometry is advocated, to replace magnetics in measuring the plasma shape and position. Collective Thomson Scattering proved successfull at diagnosing ion parameters that are normally measured by optical, beam-based diagnostics. Finally, recent proposals could enable internal, local measurements of magnetic field based on mode-conversion oblique reflectometry imaging.

On the other hand diagnostic innovations, possibly based on microwaves, might be needed in the areas of divertor, gas, neutron, radiation and some electromagnetic measurements.

\section{References}

[1] M. Shimada, D.J. Campbell, V. Mukhovatov et al., Progress in the ITER Physics Basis, Nucl. Fusion 47 (2007) S1-S413

[2] F. Turco, C.C. Petty, T.C. Luce et al., The high- $\beta_{N}$ hybrid scenario for ITER and FNSF steady-state missions, Phys. Plasmas 22 (2015) 056113

[3] C.E. Kessel, J.P. Blanchard, A. Davis et al., The Fusion Nuclear Science Facility, the Critical Step in the Pathway to Fusion Energy, Fusion Sci. Technol. 68 (2015) 225

[4] C.E. Kessel, J.P. Blanchard, A. Davis et al., Fusion Energy Systems Studies (FESS) FNSF Study Year-End Report for 2015, PPPL Report PPPL-5232 (2016), http://bp.pppl.gov/pub_report/2016/PPPL-5232-abs.html

[5] H. Zohm, C. Angioni, E. Fable et al., On the physics guidelines for a tokamak DEMO, Nucl. Fusion 53 (2013) 073019

[6] I. Cook, Materials research for fusion energy, Nat. Mater. 5 (2006) 77

[7] A.J.H. Donné, A.E. Costley, Key Issues in Diagnostics for Burning Plasma Experiments, IEEE Trans. Plasma Science 32 (2004) 177

[8] H.-J. Hartfuß, R. König, A. Werner, Diagnostics for Steady State Plasmas, Plasma Phys. Control. Fusion 48 (2006) R83

[9] A.J.H. Donné, A.E. Costley, R. Barnsley et al., Chapter 7: Diagnostics, Nucl. Fusion 47 (2007) S307

[10] G. Vayakis, E.R. Hodgson, V. Voitsenya, C.I. Walker, Chapter 12: Generic Diagnostic Issues for a Burning Plasma Experiment, Fusion Sci. Technol. 53 (2008) 699

[11] A. Weller, Diagnostics for magnetic confinement fusion research, Nucl. Instr. Meth. Phys. Res. A 623 (2010) 801

[12] A.J.H. Donné, A.E. Costley, A.W. Morris, Diagnosis for plasma control on DEMO: challenges of implementation, Nucl. Fusion 52 (2012) 074015

[13] R. Boivin, private comm. (2012) 
[14] T.J. Dolan (Ed.), Magnetic Fusion Technology (Lecture Notes in Energy), Springer (2013), Chap.11, "Plasma Diagnostics" (T.J. Dolan, A.E. Costley, J. Brotankova)

[15] T.N. Todd, Diagnostic systems in DEMO: Engineering design issues, AIP Conf. Proc. 1612 (2014) 9, Villa Monastero, Varenna (Lc), Italy, 9-13 Sept. 2013.

[16] F.P. Orsitto, R. Villari, F. Moro et al., Diagnostics and control for the steady state and pulsed tokamak DEMO, Nucl. Fusion 56 (2016) 026009

[17] Research Needs for Magnetic Fusion Energy Sciences, Report of the Research Needs Workshop (ReNeW), Bethesda, Maryland, June 8-12, 2009

[18] G. Conway, A. Stegmeir, G. Vayakis et al., ITPA Assessment of ITER Microwave Diagnostic Design Issues, Proc. 24th IAEA Fusion Energy Conference, San Diego, USA, 8-13 Oct.2012, Paper ITR/P533

[19] A. Stegmeir, G.D. Conway, E. Poli, E. Strumberger, Analysis of the ITER low field side reflectometer employing the Beam Tracing Method, Fusion Eng. Design 86 (2011) 2928

[20] G.J. Kramer, R. Nazikian, E.J. Valeo et al., 2D reflectometer modelling for optimizing the ITER low-field side X-mode reflectometer system, Nucl. Fusion 46 (2006) S846

[21] F. da Silva, A. Silva, S. Heuraux, Simulation of multiband swept reflectometry for profile evaluation on DEMO using a FDTD Maxwell fullwave code, Proc. 42nd EPS Conf. on Plasma Phys., 22-26 June 2015, Lisbon, Portugal, Paper P4.177

[22] J.X. Liu, T. Milbourne, M. Bitter et al., Alternative optical concept for electron cyclotron emission imaging, Rev. Sci. Instrum. 85 (2014) 11D802

[23] J. Liu, W. Lee, J.-E. Leem et al., Electron Cyclotron Emission Imaging on ITER with Rowland Circle Optics, 57th Annual Meeting of the APS Division of Plasma Physics, Nov. 16-20, 2015, Savannah, GA (USA), abstract JP12.109

[24] G.D. Conway, M. Hirsch, E. Doyle et al., Stray radiation protection of ITER microwave based diagnostics, Report ITER_D_33PKHG (2010)

[25] J.W. Oosterbeek, V.S. Udintsev, F. Gandini et al., Loads due to stray microwave radiation in ITER, Fusion Eng. Design 96-97 (2015) 553

[26] A. Sirinelli, N. Antonov, R. Feder et al., Design and development activities for in-vessel and in-port components of ITER microwave diagnostics, arXiv:1504.03110 (2015)

[27] V.S. Udintsev, G. Taylor, H.K.B. Pandya et al., Engineering aspects of design and integration of ECE diagnostic in ITER, EPJ Web of Conferences 87 (2015) 03006

[28] G.D. Conway, G. Vayakis, G. Hanson et al., Requirements for calibration 8 testing of ITER microwave based diagnostic front-end components, Report ITER_D_33ZRFR (2010)

[29] G.D. Conway, V. Udintsev, M.E. Austin et al., Survey and assessment of ECE and Reflectometer calibration techniques, Report ITER_D_3622CE (2011)

[30] T. Bigelow, G. Hanson, D. Bull et al., ITER ECH and LFS Reflectometer waveguide testing, 52nd Annual Meeting of the APS Division of Plasma Physics, Nov. 8-12, 2010, Chicago, IL (USA), abstract NP9.006

[31] G.R. Hanson, J.B. Wilgen, T.S. Bigelow et al., Analysis of the ITER low field side reflectometer transmission line system, Rev. Sci. Instrum. 81 (2010) 10D920

[32] L. Amicucci, A. Bruschi, P. Buratti et al., The ENEA CARM source for nuclear fusion: Project status and perspectives, Proc. 39th Int. Conf. on Infrared, Millimeter and Terahertz waves (IRMMW-THz), Tucson, AR, USA, 14-19 Sept. 2014

[33] E.W. Bryerton, D. Koller, J.L. Hesler, T.W. Crowe, A compact and reliable 200300 GHz receiver for the ITER ECE system, Proc. 39th Int. Conf. on Infrared, Millimeter and Terahertz waves (IRMMW$\mathrm{THz}$ ), Tucson, AR, USA, 14-19 Sept. 2014

[34] W. Rowan, M. Austin, J. Beno et al., Electron cyclotron emission diagnostic for ITER, Rev. Sci. Instrum. 81 (2010) 10D935

[35] M.E. Austin, H.K.B. Pandya, J. Beno et al., Conceptual Design of the ITER ECE Diagnostic - An Update, EPJ Web of Conferences 32 (2012) 03003

[36] G. Taylor, M.E. Austin, J.H. Beno et al., Status of the design of the ITER ECE diagnostic, EPJ Web of Conferences 87 (2015) 03002

[37] G. Vayakis, C.I. Walker, F. Clairet et al., Status and prospects for mm-wave reflectometry in ITER, Nucl. Fusion 46 (2006) S836

[38] M. Bornatici, R. Cano, O. De Barbieri, F. Engelman, Electron Cyclotron Emission and Absorption in Fusion Plasmas, Nucl. Fusion 23 (1983) 1153 
[39] A.G. Silva, F. da Silva, S. Heuraux, B. Gonçalves, First assessment of microwave diagnostics for DEMO, Fusion Eng. Design 96-97 (2015) 948

[40] H.-J. Hartfuss, T. Geist, Fusion Plasma Diagnostics with mm-Waves: an Introduction, Wiley-VCH (2013)

[41] A. Mase, Y. Kogi, H. Hojo et al., Progress in Microwave Diagnostics and Physics Issues in Magnetically Confined Plasmas, Fusion Sci. Technol. 51 (2007) 52

[42] N.C. Luhmann, H. Bindslev, H. Park et al., Chapter 3: Microwave Diagnostics, Fusion Sci. Technol. 53 (2008) 335

[43] A. Krämer-Flecken, Microwave and Far Infrared Diagnostics, Fusion Sci. Technol. 61 (2012) 376

[44] A. Litnovsky, V. Voitsenya, T. Sugie et al., Progress in research and development of mirrors for ITER diagnostics, Nucl. Fusion 49 (2009) 075014

[45] E.E. Mukhin, V.V. Semenov, A.G. Razdobarin et al., First mirrors in ITER: material choice and deposition prevention/cleaning techniques, Nucl. Fusion 52 (2012) 013017

[46] A. Litnovsky, M. Matveeva, A. Herrmann et al., First studies of ITER-diagnostic mirrors in a tokamak with an all-metal interior: results of the first mirror test in ASDEX Upgrade, Nucl. Fusion 53 (2013) 073033

[47] Y. Rong, C. Junling, C. Longwei et al., Cleaning of HT-7 Tokamak Exposed First Mirrors by Radio Frequency Magnetron Sputtering Plasma, Plasma Sci. Technol. 16 (2014) 1158

[48] L. Moser, L. Marot, B. Eren et al., Towards plasma cleaning of ITER first mirrors, Nucl. Fusion 55 (2015) 063020

[49] A. Maffini, A. Uccello, D. Dellasega, M. Passoni, Laser cleaning of diagnostic mirrors from tungstenoxygen tokamak-like contaminants, Nucl. Fusion 56 (2016) 086008

[50] R.E. Feder, M.Z. Youssef, ITER generic diagnostic Upper Port Plug nuclear heating and personnel dose rate assesment, Proc. 23rd IEEE/NPSS Symposium on Fusion Engineering, 2009. SOFE 2009, 1-5 June 2009, DOI: 10.1109/FUSION.2009.5226490

[51] M.R. Gilbert, S.L. Dudarev, D. Nguyen-Manh et al., Neutron-induced dpa, transmutations, gas production, and helium embrittlement of fusion materials, J. Nucl. Mater. 442 (2013) S755

[52] I. Bolshakova, S. Belyaev, M. Bulavin et al., Experimental evaluation of stable long term operation of semiconductor magnetic sensors at ITER relevant environment, Nucl. Fusion $\mathbf{5 5}$ (2015) 083006

[53] Neutron Transmutation Doping of Silicon at Research Reactors, Report IAEA-TECDOC-1681, International Atomic Energy Agency, Vienna, 2012 ISBN 978-92-0-130010-2

[54] S.J. Zinkle, E.R. Hodgson, T. Shikama, Proc. 9th IEA Workshop on Radiation Effects in Ceramic Insulators, Cincinnati, Ohio, May 8 9, 1997, Oak Ridge National Lab Report ORNL/M-6068 (1997)

[55] K. Shiiyama, M.M.R. Howlader, S.J. Zinkle et al., Electrical conductivity and current-voltage characteristics of alumina with or without neutron and electron irradiation J. Nucl. Mater. 258-263 (1998) 1848

[56] Fission-reactor-radiation-tests of MI-cables and magnetic coils for fusion burning plasma diagnostics, T. Shikama, S. Yamamoto, R. Snider et al., Fusion Eng. Design 51-51 (2000) 171

[57] S.J. Zinkle, E.R. Hodgson, Radiation-induced changes in the physical properties of ceramic materials, J. Nucl. Mater. 191-194 (1992) 58

[58] J.-L. Leray, S. Bazzoli, O. Duhamel et al., Experimental evidence of Radiation-Induced Conductivity (n-RIC) in PE and PTFE materials caused by ultra-short pulses of 14 MeV neutrons in inertial confinement fusion, Proc. 3rd Int. Conf. on Advancements in Nuclear Instrumentation Measurement Methods and Applications (ANIMMA), 23-27 June 2013, DOI: 10.1109/ANIMMA.2013.6727962

[59] G. Vayakis, C. Walker, Magnetic diagnostics for ITER/BPX plasmas (invited), Rev. Sci. Instrum. 74 (2003) 2409

[60] G. McKee, priv. comm. (2016)

[61] A. Gorshkov, D. Orlinski, V. Sannikov, et al., Measurement of the Radiation Resistant Fused Quartz Radioluminescence Spectral Intensity Under Irradiation in the Pulse Nuclear Reactor, J. Nucl. Mater. 273 (1999) 271

[62] A.E. Costley, D.J. Campbell, S. Kasai et al., ITER RED: Auxiliary Systems: Plasma Diagnostics, Fusion Eng. Design 55 (2001) 331

[63] I. Duran, M. Hron, J. Stöckel et al., Stability of the Hall sensors performance under neutron irradiation, Proc. 12th Int. Congress Plasma Physics, Nice, 2004.

[64] C.D. Amaden, Fusion neutron damage to a charge coupled device camera, Thesis 1997-09, Naval Postgraduate School, Monterey, CA (1997) 
[65] C.H. Skinner, W. Blanchard, J. Kamperschroer et al., Measurements of tritium retention and removal on the Tokamak Fusion Test Reactor, J. Vac. Sci. Technol. A 14 (1996) 3267

[66] I.H. Coffey, R. Barnsley and JET EFDA Contributors, First tritium operation of ITER-prototype VUV spectroscopy on JET, Rev. Sci. Instrum. 75 (2004) 3737

[67] T. Nishitani, G. Vayakis, M. Yamauchi et al., Radiation-induced thermoelectric sensitivity in the mineral-insulated cable of magnetic diagnostic coils for ITER, J. Nucl. Mater. 329 (2004) 1461

[68] G. Vayakis, T. Sugie, T. Kondoh et al., Radiation-induced thermoelectric sensitivity (RITES) in ITER prototype magnetic sensors, Rev. Sci. Instrum. 75 (2004) 4324

[69] P. Moreau, P. Defrasne, E. Joffrin et al., A magnetic diagnostic on Tore Supra, Rev. Sci. Instrum. 74 (2003) 4324

[70] R.K. Kanev, C.D. Boley, D.E. Post, Penetration of energetic neutral beams into fusion plasmas, Nucl. Fusion 29 (1989) 2125

[71] F.D. Halpern, A.H. Kritz, G. Bateman, Predictive simulations of ITER including neutral beam driven toroidal rotation, Phys. Plasmas 15 (2008) 062505

[72] D.J. Rej, I. Henins, R.J. Fonck, Y.J. Kim, Intense diagnostic neutral beam development for ITER, Rev. Sci. Instrum. 63 (1992) 4934

[73] G.R. McKee, R.J. Fonck, D.K. Gupta et al., High sensitivity beam emission spectroscopy for core plasma turbulence imaging (invited), Rev. Sci. Instrum. 77 (2006) 10F104

[74] R.J. Fonck, Charge exchange recombination spectroscopy as a plasma diagnostic tool (invited), Rev. Sci. Instrum. 56 (1985) 885

[75] R.C. Wolf, A. Bock, O.P. Ford et al., Motional Stark Effect measurements of the local magnetic field in high temperature fusion plasmas, JINST 10 (2015) P10008

[76] L. Giannone, D. Queen, F. Hellman, J.C. Fuchs, Prototype of a radiation hard resistive bolometer for ITER, Plasma Phys. Control. Fusion 47 (2005) 2123

[77] B.J. Peterson, E.A. Drapiko, D. Seo, N. Ashikawa, , Comparison of Au and Pt Foils for an Imaging Bolometer, Plasma Fusion Res. 5 (2010) 035

[78] V. Voitsenya, A.E. Costley, A.J.H. Donné et al., Diagnostic first mirrors for burning plasma experiments (invited), Rev. Sci. Instrum. 72 (2001) 475

[79] R.W. Moir, Grazing incidence liquid metal mirrors (GILMM) for radiation hardened final optics for laser inertial fusion energy power plants, Fusion Eng. Design 51-52 (2000) 1121

[80] P.F. Goldsmith, Quasioptical Systems: Gaussian Beam Quasioptical Propagation and Applications, Wiley-IEEE Press (1998)

[81] S. Adachi, The Handbook on Optical Constants of Metals: In tables and Figures, World Scientific (2012)

[82] G. Cima, R.V. Bravenec, A.J. Wootton et al., Core temperature fluctuations and related heat transport in the Texas Experimental Tokamak-Upgrade, Phys. Plasmas 2 (1995) 720

[83] A.E. White, L. Schmitz, W.A. Peebles et al., Simultaneous measurement of core electron temperature and density fluctuations during electron cyclotron heating on DIII-D, Phys. Plasmas 17 (2010) 020701

[84] C. Sung, A.E. White, J.H. Irby et al., Design of a correlation electron cyclotron emission diagnostic for Alcator C-Mod Rev. Sci.Instrum. 83 (2012) 10E311

[85] W.F. Bergerson, P. Xu, J.H. Irby et al., Far-infrared polarimetry diagnostic for measurement of internal magnetic field dynamics and fluctuations in the C-MOD Tokamak (invited), Rev. Sci. Instrum. 83 (2012) 10E316

[86] C. Fuchs, H.J. Hartfuss, Line integrated density measurements based on CottonMouton polarimetry, Rev. Sci. Instrum. 70 (1999) 722

[87] S.E. Segre, A review of plasma polarimetry - theory and methods, Plasma Phys. Control. Fusion 41 (1999) R57

[88] L.L. Lao, J.R. Ferron, R.J. Groebner et al., Equilibrium Analysis of Current Profiles in Tokamaks, Nucl. Fusion 30 (1990) 1035

[89] J. Santos, L. Giumarãis, M. Zilker, W. Treutterer, M. Manso, Reflectometry-based plasma position feedback control demonstration at ASDEX Upgrade, Nucl. Fusion 52 (2012) 032003

[90] E. Ricardo, P. Varela, A. Silva, B. Goncalves, Assessment and performance optimization of the ITER plasma position reflectometry in-vessel oversized waveguide bends, Fusion Eng. Design 98-99 (2015) 1593 
[91] G. Giruzzi, I. Fidone, M.J. Marchã, Diagnosis of the current-carrying lower-hybrid tail, Plasma Phys. Control. Fusion 31 (1989) 1921

[92] S. Preische, P.C. Efthimion, S.M. Kaye, Radially localized measurements of superthermal electrons using oblique electron cyclotron emission, Phys. Plasmas 3 (1996) 4065

[93] S. Preische, P.C. Efthimion, S.M. Kaye, Oblique electron cyclotron emission for electron distribution studies (invited), Rev. Sci. Instrum. 68 (1997) 409

[94] E. de la Luna, V. Krivenski, G. Giruzzi et al., Impact of bulk non-Maxwellian electrons on electron temperature measurements (invited), Rev. Sci. Instrum. 74 (2003) 1414

[95] A. Simonetto, C. Sozzi, S. Garavaglia et al., A fast multichannel Martin-Puplett interferometer for electron cyclotron emission measurements on JET, Rev. Sci. Instrum. 82 (2011) 113506

[96] K. Kato, I.H. Hutchinson, Nonthermal Electron Velocity Distribution Measured by Electron Cyclotron Emission in Alcator C Tokamak, Phys. Rev. Lett. 56 (1986) 340

[97] K. Kato, I.H. Hutchinson, Diagnosis of mildly relativistic electron velocity distributions by electron cyclotron emission in the Alcator C tokamak, Phys. Fluids 30 (1987) 3809

[98] T.C. Luce, P.C. Efthimion, N.J. Fisch, Suprathermal electron distribution measurements from polarized electron cyclotron emission, Rev. Sci. Instrum. 59 (1988) 1593

[99] A.G. Sitenko, Yu.A. Kirochkin, Scattering and Transformation of Waves in a Magnetoactive Plasma, Soviet Phys. Usp. 9 (1966), 430

[100] R.E. Aamodt, D.A. Russell, Alpha Particle Detection by Electromagnetic Scattering off of Plasma Fluctuations, Nucl. Fusion 32 (1992), 745

[101] F. Orsitto, G. Giruzzi, Review of possibilities for a collective Thomson scattering system on ITER, Rev. Sci. Instrum. 68 (1997) 686

[102] F. Volpe, Scattering Collettivo di Onde Millimetriche per la Misura della Temperatura Ionica sul Tokamak FTU, "Laurea" Dissertation, Univ. of Pisa, Italy, March 1998, available at http://www.ipp.mpg.de/ frv/Publ/Lau.pdf, page 34-36

[103] H. Bindslev, S.K. Nielsen, L. Port et al., Fast-Ion Dynamics in the TEXTOR Tokamak Measured by Collective Thomson Scattering, Phys. Rev. Lett. 97 (2006) 205005

[104] E.V. Suvorov, V. Erckmann, E. Holzhauer et al., Ion temperature and beam-driven plasma waves from collective scattering of gyrotron radiation in W7-AS, Plasma Phys. Control. Fusion 37 (1995) 1207

[105] S. Catiglioni, M. Lontano, U. Tartari, Effects of the ohmic current on collective scattering spectra, Plasma Phys. Control. Fusion 35 (1993) 1395

[106] H. Bindslev, S.K. Nielsen, L. Porte et al., Fast-ion dynamics in the TEXTOR tokamak measured by collective Thomson scattering, Plasma Phys. Control. Fusion 49 B551 (2007)

[107] M. Salewski, O. Asunta, L.-G. Eriksson et al., Comparison of collective Thomson scattering signals due to fast ions in ITER scenarios with fusion and auxiliary heating, Plasma Phys. Control. Fusion 51 (2009) 035006

[108] F. Orsitto, A. Brusadin, Yu. Brodsky et al., Characterization and Preliminary Results of the Collective Thomson Scattering (CTS) System on FTU Tokamak, Rev. Sci. Instrum. 70 (1999) 1158

[109] M. Stejner, S.B. Korsholm, S.K. Nielsen et al., Measurements of plasma composition in the TEXTOR tokamak by collective Thomson scattering, Plasma Phys. Control. Fusion 54 (2012) 015008

[110] Equipe TFR, Tokamak Plasma Diagnostics, Nucl. Fusion 18 (1978) 647

[111] H. Soltwisch, Current density measurements in Tokamak devices, Plasma Phys. Control. Fusion 34 (1992) 1669

[112] R.C. Wolf, J. O'Rourke, A.W. Edwards, M. Von Hellermann, Comparison of Poloidal Field Measurements on JET, Nucl. Fusion 33 (1993) 663

[113] K.W. Gentle, Diagnostics for magnetically confined high-temperature plasmas, Rev. Mod. Phys. 67 (1995) 809

[114] A.J.H. Donné, Diagnostics for current density and radial electric field measurements: overview and recent trends, Plasma Phys. Control. Fusion 44 (2002) B137

[115] V.Shevchenko, G.Cunningham, A.Gurchenko et al., Development of Electron Bernstein Wave Research in MAST, Fusion Sci. Technol. 52 (2007) 202

[116] F. Volpe, A Spinning Mirror for Fast Angular Scans of EBW Emission for Magnetic Pitch Profile Measurements, Rev. Sci. Instrum. 81 (2010) 10D905 
[117] S.J. Freethy, B.K. Huang, V.F. Shevchenko, R.G.L. Vann, Lensless passive and active microwave imaging on MAST, Plasma Phys. Control. Fusion 55 (2013) 124010

[118] O. Meneghini, F.A. Volpe, Full-wave feasibility study of anti-Radar diagnostic of magnetic field based on $O$-X mode conversion and oblique reflectometry imaging, Rev. Sci. Instru. 87 (2016) 11E120

[119] K. McCormick, P. Grigull, R. Burhenn et al. New Advanced Operational Regime on the W'-AS Stellarator, Phys. Rev. Lett. 89 (2002) 015001

[120] A. Costley, priv. comm. (2004)

[121] H. Sugai, Mode Conversion and Local Heating below the Second Electron Cyclotron Harmonic, Phys. Rev. Lett. 47 (1981) 1899

[122] E. Mazzucato, D.R. Smith, R.E. Bell et al., Short-Scale Turbulent Fluctuations Driven by the Electron-Temperature Gradient in the National Spherical Torus Experiment, Phys. Rev. Lett. 101 (2008) 075001

[123] X.L. Zou, L. Colas, M. Paume et al., Internal Magnetic Turbulence Measurement in Plasma by Cross Polarization Scattering, Phys. Rev. Lett. 75 (1995) 1090

[124] R.G.L. Vann, K.J. Brunner, R. Ellis et al., Preliminary measurements of the edge magnetic field pitch from 2-D Doppler backscattering in MAST and NSTX-U (invited), Rev. Sci. Instrum. 87 (2016) 11D902

[125] S.P. Gerhardt, E. Fredrickson, L. Guttadora et al., Techniques for the measurement of disruption halo currents in the National Spherical Torus Experiment, Rev. Sci. Instrum. 82 (2011) 103502

[126] E. Lazzaro, I. Proverbio, F. Nespoli et al., Transport and effects of ferromagnetic dust in a tokamak with a metallic vessel, Plasma Phys. Control. Fusion 54 (2012) 124043 